\title{
A novel STS mutation and an Xp22.3 microdeletion from one Chinese family with $\mathrm{X}$-linked ichthyosis
}

\author{
Yufeng Huang \\ Wuhan Children's Hospital \\ Sukun Luo \\ Wuhan Children's Hospital \\ Peiwei Zhao \\ Wuhan Children's Hospital \\ Li Tan \\ Wuhan Children's Hospital \\ Guili Fu \\ Wuhan Children's Hospital \\ Xuelian He ( $\nabla$ hexuelian2013@hotmail.com ) \\ Wuhan Women and Children Medical Care Center https://orcid.org/0000-0002-6275-1219
}

\section{Research article}

Keywords: X-linked ichthyosis, exome sequencing, STS, mutation, deletion

Posted Date: July 15th, 2020

DOI: https://doi.org/10.21203/rs.3.rs-23257/v1

License: (c) (1) This work is licensed under a Creative Commons Attribution 4.0 International License.

Read Full License 


\section{Abstract}

Background X-linked ichthyosis (XLI, OMIM\# 308100) is a relatively common type of skin disorder, characterized by widespread, dark brown, polygonal scales and generalized dryness. Most patients (90\%) are contributed to genomic deletion of the entire STS (steroid sulfatase) gene encoding steroid sulfatase, with the remaining cases being caused by point mutations or partial deletions. The patient carrying genomic deletion comprising the entire STS gene and adjacent genes often manifests neurological symptoms.

Methods Herein, we reported a large four-generation Chinese family with 5 individuals with XLI with/ without mental delay. Genetic analysis was conducted by whole exome sequencing and confirmed by Sanger sequencing and quantitative Real-time PCR.

Results These patients present generalized dryness and scaling of the skin with dark scales of the skin on trunk, and limbs. Two pathogenic variants, a novel STS mutation (c.1532A > G, p.E511G, NM_000351) and a 2 Mb genomic microdeletion at Xp22.3 $(6,451,785-8,434,424)$ embracing entire STS gene were identified in this family. In addition, an individual with Xp22.3 deletion has mental delay.

Conclusion Our study expands the genetic profile of the STS gene in XLI patients and highlights the clinical application of exome sequencing.

\section{Background}

The ichthyoses are a heterogeneous group of dermatological conditions characterized by widespread, dark brown, polygonal scales and generalized dryness. Cutaneous manifestations are frequently present few weeks after birth, usually do not improve with age but affect a person throughout life [1]. The majority of ichthyoses is inherited and can be pinpointed to characteristic genetic mutations. X-linked recessive ichthyosis (XLI, OMIM\#308100) is a relatively common type of ichthyosis with an estimated prevalence ranging from 1:1500 to 1:6000 worldwide[2]. XLI was first described in 1927 by Lundborg[3]. It may occur solely as a skin disorder or may be associated with other physical findings such as corneal opacities (up to $50 \%$ of cases), cryptorchidism ( $20 \%$ of cases), chondrodysplasia punctata, and nephritic syndrome [4]. X-linked ichthyosis is caused by the deletion of or mutations in the STS (steroid sulfatase) gene encoding steroid sulfatase. The STS gene is located on chromosome Xp22.3, containing 10 exons and spans about $146 \mathrm{~kb}[5]$. No homologous sequences were detected on the $Y$ chromosome[6] and nonX-inactivation was reported in this gene[7]. Most patients (85\%-90\%) have a complete or partial deletion of the STS gene, while point mutations within STS gene account for about $10 \%$ of cases[8]. The protein encoded by STS gene is a steroid sulfatase enzyme, a membrane-bounding protein present in most organs and tissues including the skin, breast, blood and brain[9]. Patients with mutations involving solely the STS gene may present with attention deficit hyperactivity disorder (ADHD), while those with deletions including neighboring genes such as neuroligin 4 (NLGN4) may be associated with multiple neurodevelopmental disorders, including mental retardation, autism spectrum disorders (ASDs), ADHD, 
and seizures[4]. In addition, a recent study showed adult males with XLI disease-causing deletions are apparently at increased risk of cardiac arrhythmias and self-reported mood problems[10].

In this study, we report a four-generation Chinese family with XLI and identified a novel hemizygote point mutation (c.1532 A > G, p.E511G, NM_000351) in one branch and a recurent $2 \mathrm{Mb}$ genomic deletion (Xp22.3: 6,451,785-8,434,424) comprising the entire STS gene in other branch. These variants were absent in healthy family members and cosegregated with XLI in this pedigree. Our study extended the knowledge of mutation spectrum in XLI patients and highlighted the clinical application of exome sequencing.

\section{Methods}

\section{Subjects}

The XLI family in this study has 47 members across four generation and 5 patients were diagnosed with ichthyosis. The family pedigree is shown in Fig. 1A. Written informed consents were obtained from all subjects or the patient (III:6)'s guardian, and the study was approved by the institutional review board of Wuhan Children's Hospital, Tongji Medical College, Huazhong University of Science \& Technology (2019011). All experiments were performed in accordance with the Declaration of Helsinki.

\section{Whole-exome Sequencing}

Whole exome sequencing was carried out by a commercial company (Chigene, Beijing, China) following standard experimental procedures. The exome was captured using IDT the xGen Exome Research Panel v1.0 Kit. The high-throughout sequencing was based on Illumina NovaSeq 6000 (Illumina, San Diego, CA, USA). The read length of the sequencing was paired-end $150 \mathrm{bp}$, and the read depth was 120x. Trio whole exome sequencing was carried out, respectively, on the proband family (III:1, II:1, and II:2) and his cousin family (III:6, II:7, and II:8).

\section{Bioinformatics Analysis}

The possible effects of the variant on the function and structure of protein, and likelihood of pathological damage were analyzed by SIFT, MutationTaster, PolyPhen2-HDIV, Provean, PolyPhen2-HVAR, M-CAP and REVEL. The sequences of steroid sulfatase protein from zebrafish, trubripes, Mmulatta, human and chimpanzee were aligned using Clustal Omega Sanger sequencing.

\section{Segregation Analysis}

Direct Sanger sequencing was carried out in III:1, II:1, II:2, III:3, III:5 to identify the potential diseasecausing mutations. Real-time PCR was used to determine the copy number of STS gene of II:7, II:8, III:6, 
III:7, III:9, III:11, III:19, and of PNPLA4 gene of II:8, III:6, III:19. All primers were shown in Table 1.

Table 1

Sequences for primers for PCR and real-time RT-PCR analyses

\begin{tabular}{|lll|}
\hline Primers & Forward & \multicolumn{1}{c|}{ Reverse } \\
\hline $\begin{array}{l}\text { Gene or } \\
\text { fragments }\end{array}$ & GCAGCATAATTTCCGCATCACTT & CCACAGAAGGCATAAACCTACCA \\
STS-1Q & CCTCACATTATCTGCCCAAGCAC & TTGAAGCAGGCCAATCCTACTCA \\
STS-5Q & CCCTTTACATCACGGCTTCAATTATT & AGTAGCCCCAGACAATTGAGTG \\
\hline STS-10Q & CTTCTGTTTCGGGAGTTATGTCACC & CATGAAAACTGATCGGGCACCT \\
\hline PNPLA4 & GCGGGATCGTTGGTTGCTTCT & GCTGGTGTTTGCCTTTGGGTCTT \\
\hline CYBB & AGTCACTCTGCTCCCTTTCC & CGACAGACTGGCAAGAGAATC \\
\hline 36B4 & CAGCAAGTGGGAAGGTGTAATCC & CCATTCTATCATCAACGGGTACAA \\
\hline
\end{tabular}

\section{Results}

\section{Clinical information}

The proband (III:1, male, 32 years old, shown in Fig. 1A) came to our genetic clinic for counseling as his sister (III:3) was pregnant. The proband complained scattered ichthyosis all over the body, which became more severe in winter with dry and cold weather (shown in Fig. 1B). Our patient has one aunt and three uncles from his mother's side, and his aunt's son (III:5) also displayed similar symptom. In addition to the classic feature of ichthyosis, the son (Ш:6, 37 years old) of his youngest uncle $(\Pi: 7)$ exhibited intellectual disability and speech delay, with a history of febrile convulsion (shown in Fig. 1A). Three daughters (III:7, III:9, III:11) of this uncle did not show signs of ichthyosis. Interestingly, the branch of his aunt (II8)'s family also have another two individuals with ichthyosis and barriers to effective communication ( $П: 11$ and Ш:19), with normal intelligence (Fig. 1A).

\section{Whole-exome Sequencing, Bioinformatics And Segregation Analysis}

Through whole exome sequencing, after the filtering steps, a solitary heterozygous missense variant, c.1532A > G (p.E511G), in exon 10 of the STS gene (NM_000351) was identified in the proband III:1 based on sequences from NCBI database. Further Sanger sequencing revealed that individual III:5 was hemizygous while II:1, П:5 and III:3 were carriers (shown in Fig. 1C). This mutation is predicted to be pathogenic by bioinformatics tools and conserved (Fig. 1D) among different species, and it is not observed in other family members. The c.1532A > G mutation has not been reported previously, and is not present in any public genomic variants database, including Human Genome Mutation Database, 
1000Genomes, ESP6500, ExAC Browser (exome aggregation consortium), and COSMIC (the catalog of somatic mutations in cancer), indicating that c.1532A $>\mathrm{G}$ in STS is a novel mutation that may cause ichthyosis.

As the mutation, c.1532A > G (p.E511G), was not detected in the individual Ш:6 with ichthyosis, intellectual disability and speech delay, a trio whole-exome sequencing was performed. After data analysis, a maternal genomic deletion of $1.98 \mathrm{Mb}$ in chromosome Xp22.31 $(6,451,785-8,434,424)$ comprising the entire STS gene and 6 neighboring gene (HDHD1, PNPLA4, VX,VCX2, VCX3A, and VCX3B) (Fig. 1E), and a de novo duplication of $2.59 \mathrm{Mb}$ of in chromosome 11q14.3 $(89,947,185-92,538,515)$ containing two protein-coding genes (FAT3 and CHORDC1) were observed. Further RT-PCR was performed on mRNA from the patient III:6 and his siblings (III:7, III:9, and III:11) using primers for STS and one of deleted neighboring gene, PNPLA4. RT-PCR showed that the STS and PNPLA4 gene were absent in Ш:6 but not in his siblings. In addition, individual III:19, who had similar ichthyoses with normal intelligence, was also tested and found to the deletion of STS and PNPLA4 gene. The individual II:11, who had same symptom as individual III:19, was not genetically tested, as blood sample was not available. All these RTPCR results were shown in Fig. $1 \mathrm{~F}$ and $1 \mathrm{G}$. Taken together, two genetic variants, point mutation (c.1532A $>$ G, p.E511G) and a maternal genomic deletion of $1.98 \mathrm{Mb}$ in chromosome Xp22.31, were observed in the family.

\section{Discussion}

In this study, we reported two variants from one large family, one is a novel missense mutation in the proband with ichthyosis, and the other one is a maternal deletion in Xp22.31 in his cousin (the son of his uncle from his mother's side) with ichthyosis and disability. XLI is a recessive disorder and it almost exclusively affects males. Four XLI female cases have been described, three of these patients were offspring of an affected father and a carrier mother[11, 12], and one patient was adopted girl who was homozygous nonsense mutation in the STS gene[8].

To date, in addition intragenic large deletions, a total of 29 STS variants, including the variant (c.1532A > G, p.E511G) reported in this study, have been identified. As shown in Fig. 2 and Table 2, of the 29 variants, 5 are truncating, 3 are frameshift, 20 are missense, and 1 is a deletion involving one amino acid, and it seems that exon 7 and 8 are the hotspot variation region. 
Table 2

Point mutations in the STS gene reported to date in the literature/ database

\begin{tabular}{|c|c|c|c|c|}
\hline No. & Sequence change & Amino acid change & exon & Reference/database \\
\hline 1 & c. $184 \mathrm{G}>\mathrm{T}$ & G62R & 3 & Clinvar \\
\hline 2 & c. $261 \mathrm{C}>\mathrm{G}$ & Y87X & 3 & del Refugio Rivera Vega et [21 al[21] \\
\hline 3 & c. $268 \mathrm{C}>\mathrm{T}$ & R90X & 3 & Winge et al[22] \\
\hline 4 & c. $287 G>A$ & W96X & 4 & Afzal etal[23] \\
\hline 5 & c. $323 \mathrm{C}>\mathrm{T}$ & S108L & 4 & Diociaiuti etal[9] \\
\hline 6 & c. $452 C>G$ & P151R & 5 & Diociaiuti et al[24] \\
\hline 7 & c. $494 \mathrm{C}>\mathrm{T}$ & T165I & 5 & Liao et al[25] \\
\hline 8 & c.529_532del4insAG & p.V177Sfs81 & 5 & Takeichi et al[26] \\
\hline 9 & c. $1022 \mathrm{C}>\mathrm{T}$ & S341L & 7 & Basler et al[27] \\
\hline 10 & c. $1030 \mathrm{G}>\mathrm{A}$ & G344R & 7 & Diociaiuti1 etal[9] \\
\hline 11 & c. $1032 C>T$ & G344X & 7 & Morita et al[28] \\
\hline 12 & c.1046_1048delAAG & E349del & 7 & Valdes-Flores et al[29] \\
\hline 13 & c. $1049 \mathrm{~T}>\mathrm{G}$ & V350G & 7 & Oyama et al[30] \\
\hline 14 & c. $1075 \mathrm{G}>\mathrm{A}$ & G359R & 7 & Diociaiuti et al[24];Oyama et al[30] \\
\hline 15 & c. $1099 \mathrm{G}>\mathrm{A}$ & G367R & 8 & Wei et al[31] \\
\hline 16 & c. $1114 \mathrm{~T}>\mathrm{A}$ & W372R & 8 & Basler et al, Alperin et al[27] \\
\hline 17 & c. $1115 G>C$ & W372S & 8 & Alperin et al[5] \\
\hline 18 & c. $1138 \mathrm{G}>\mathrm{C}$ & G380R & 8 & Oyama et al[32] \\
\hline 19 & c. $1165 \mathrm{C}>\mathrm{T}$ & Q389X & 8 & Oyama et al[32] \\
\hline 20 & c.1213_1214insTC & P405Lfs408* & 8 & Murtaza etal[33] \\
\hline 21 & c. $1256+1 G>T$ & R419Sfs427* & 8 & Alperin et al[5] \\
\hline 22 & c. $1331 \mathrm{~A}>\mathrm{G}$ & H444R & 9 & Alperin et al[5] \\
\hline 23 & c. $1337 \mathrm{G}>\mathrm{A}$ & C446Y & 9 & Basler et al[27] \\
\hline 24 & c. $1360 \mathrm{C}>\mathrm{T}$ & R454C & 9 & Gonzalez-Huerta et al[34] \\
\hline 25 & c. $1361 G>A$ & $\mathrm{R} 454 \mathrm{H}$ & 9 & Valdes-Flores et al[29] \\
\hline 26 & c. $1393 A>G$ & K465E & 10 & Trevisson et al[35] \\
\hline 27 & c. $1532 A>G$ & E511G & 10 & This study \\
\hline
\end{tabular}




\begin{tabular}{|lllll|}
\hline No. & Sequence change & Amino acid change & exon & Reference/database \\
\hline 28 & c.1679A $>$ C & Q560P & 10 & Sugawara et al[12] \\
\hline 29 & c.1679A > G & Q560R & 10 & Goodwin et al[36] \\
\hline
\end{tabular}

$\mathrm{XLI}$ is characterized by scaly skin on the scalp, trunk and limbs, aggravates in winter and dry climates and alleviate in summer $[13,14]$, which is consistent with the clinical features of our patient and the affected individuals in his family. Previous studies demonstrated that, in addition to skin problems, XLI patients with Xp22.31 deletion usually present with contiguous gene deletion symptoms[15], including short stature(SHOX), Kallmann syndrome( $K A L)$, X-linked chondrodysplasia punctata (ARSE) and X-linked ocular albinism(OA1). In addition, neurodevelopmental conditions, including ADHD, autism, and mental retardation, were also observed in patients with XLI[16, 17]. Recently, a study showed adult males with XLI disease-causing deletions are apparently at increased risk of cardiac arrhythmias and self-reported mood problems[10], and altered basal ganglia structure was observed in these patients[10]. In this pedigree in the present study, the patient III:6, who had ichthyosis, mental retardation, delayed speech, and history of febrile convulsion, was found to harbor a Xp22.31 deletion of $1.98 \mathrm{Mb}$, and the patient has not yet showed heart rhythm problems at the age of 35 years old. Follow-up should be done by regular examination. In addition to STS gene, the deleted region contains 6 neighboring gene (HDHD1, PNPLA4, $V X, V C X 2, V C X 3 A$, and $V C X 3 B$ ). The $V C X 3 A$ gene has been reported to be necessary to maintain normal intellectual development and proposed to be responsible for mental retardation in some XLI individuals[18]. However, highly variable phenotypes were observed in individuals with VCX3A deletions, which also happened in our study. Based on the RT-PCR results, it is likely that two patients (II:11 and Ш:19) has Xp22.31 deletion, however they did not present mental retardation but with barriers to effective communication, which further showed that the cognitive behavioral syndromes vary from normal intelligence to mental retardation, even with same size of deletion. Previous report also showed that no difference in deletion size of fragment including $V C X-A$ for $X L I$ patients with and without mental retardation[18]. We also noted that the patient (II:6) harbored a de novo duplication of $2.59 \mathrm{Mb}$ in $11 \mathrm{q} 14.3$ containing 12 genes, inducing two protein-coding genes (FAT3 and $C H O R D C 1$ ), 8 noncoding genes (DISC1FP1, NDUFB11P1, OSBPL9P2, OSBPL9P3, PGAM1P9, RPL7AP57, RPS3AP42 and TUBB4BP4) and microRNAs(MIR1261 and MIR4490), it is unclear that whether this duplication is correlated to mental retardation in this patient. Thus, the detailed molecular mechanism underlying mental retardation in patients with XLI and Xp22.31 deletion need further investigation.

Both Xp22.31 deletion and point mutations in the STS gene were also correlated with ADHD, suggesting that STS deficiency has a curial role in the pathogenesis of inattentive/hyperactivity symptoms. Mouse models also support this hypothesis[19]. In addition to ADHD, other symptoms, such as short stature, epilepsy, bone density reduction, and cryptorchidism, were observed in two siblings [20]. However, our XLI patients with point mutation in the STS gene did not display any symptoms of ADHD or other related cognitive behavioral problems. 


\section{Conclusion}

Through high throughput sequencing of a four-generation Chinese family, we identified a novel missense mutation of STS gene in a patient with ichthyosis and a $2 \mathrm{Mb}$ Xp22.3 deletion in his cousin with ichthyosis and mental retardation, expanding the mutation spectrum of XLI. Meanwhile, the study also demonstrates that the genotype-phenotype correlation of $\mathrm{XLI}$ is very complicated, further study is needed. Finally, our study emphasizes the importance of whole exome sequencing for genetic diagnosis in rare diseases.

\section{Abbreviations}

$X L I$

X-linked ichthyosis

ADHD

attention deficit hyperactivity disorder

NLGN4

neuroligin 4

ASD

autism spectrum disorder

SIFT

sorting intolerant from tolerant

\section{Declarations}

\section{Ethics approval and consent to participate}

The study was done after obtaining the ethics approval from the institutional review board of Wuhan Children's Hospital, Tongji Medical College, Huazhong University of Science \& Technology(approval number 2019011), and with the patient (proband) or the guardian of one patient who has mental delay.

\section{Consent for publication}

Written informed consent for publication of clinical details and/or clinical images was obtained from all of the participants or the guardian of one patient who has mental delay.

\section{Availability of data and material}

The authors confirm that the data supporting the findings of this study are available within the article.

\section{Competing interests}

The authors declare that they have no competing interests. 


\section{Funding}

This work was supported by the grants of Wuhan Municipal Health Commission (NO.WX17Z12 from Xuelian He, WX19C19 from Yufeng Huang, WX14A06 from Peiwei Zhao); Youth Program of National Natural Science Foundation of China (NO.81700302) from Yufeng Huang; Natural Science Foundation of Hubei Province (2017CFB322) from Sukun Luo.

\section{Authors' contributions}

Study concepts: $\mathrm{XH}, \mathrm{YH}$

Study design: $\mathrm{YH}$

Literature reserch: SL

Clinical information collection: GF, YH

Data acquisition: $\mathrm{YH}, \mathrm{PZ}$

Data analysis/interpretation: LT

Manuscript preparation: YH

Manuscript editing/ revision/review: XH

All authors have read and approved the manuscript

\section{Acknowledgements}

We thank all patients for their participation in the study.

\section{References}

1. Huang JW, Tang N, Li WG, Li ZT, Luo SQ, Li JW, Huang J, Yan TZ: [Identification of gene mutation and prenatal diagnosis in a family with X-linked ichthyosis]. Zhongguo Dang Dai Er Ke Za Zhi 2016, 18(11):1136-1140.

2. Zhang $M$, Huang $H$, Lin N, He S, An G, Wang Y, Chen M, Chen L, Lin Y, Xu L: X-linked ichthyosis: Molecular findings in four pedigrees with inconspicuous clinical manifestations. J Clin Lab Anal 2020:e23201.

3. Schlammadinger J, Meyer JC, Vajda I, Szabo G: X-linked recessive ichthyosis. Reinvestigation of a family first described in 1928. Dermatologica 1987, 175(5):217-223. 
4. Malik A, Amer AB, Salama M, Haddad B, Alrifai MT, Balwi MA, Davies W, Eyaid W: X-linked ichthyosis associated with psychosis and behavioral abnormalities: a case report. J Med Case Rep 2017, 11(1):267.

5. Alperin ES, Shapiro LJ: Characterization of point mutations in patients with X-linked ichthyosis. Effects on the structure and function of the steroid sulfatase protein. J Biol Chem 1997, 272(33):20756-20763.

6. Ballabio A, Parenti G, Carrozzo R, Sebastio G, Andria G, Buckle V, Fraser N, Craig I, Rocchi M, Romeo G et al: Isolation and characterization of a steroid sulfatase cDNA clone: genomic deletions in patients with X-chromosome-linked ichthyosis. Proc Natl Acad Sci U S A 1987, 84(13):4519-4523.

7. Cavenagh A, Chatterjee S, Davies W: Behavioural and psychiatric phenotypes in female carriers of genetic mutations associated with X-linked ichthyosis. PLoS One 2019, 14(2):e0212330.

8. Diociaiuti A, Angioni A, Pisaneschi E, Margollicci M, Boldrini R, Alesi V, Novelli A, Zambruno G, El Hachem M: Next Generation Sequencing Uncovers a Rare Case of X-linked Ichthyosis in an Adopted Girl Homozygous for a Novel Nonsense Mutation in the STS Gene. Acta Derm Venereo/2019, 99(9):828-830.

9. Diociaiuti A, Angioni A, Pisaneschi E, Alesi V, Zambruno G, Novelli A, El Hachem M: X-linked ichthyosis: Clinical and molecular findings in 35 Italian patients. Exp Dermato/ 2019, 28(10):11561163.

10. Brcic L, Underwood JF, Kendall KM, Caseras X, Kirov G, Davies W: Medical and neurobehavioural phenotypes in carriers of X-linked ichthyosis-associated genetic deletions in the UK Biobank. J Med Genet 2020.

11. Wells RS, Kerr CB: Genetic classification of ichthyosis. Arch Dermatol 1965, 92(1):1-6.

12. Sugawara T, Shimizu H, Hoshi N, Fujimoto Y, Nakajima A, Fujimoto S: PCR diagnosis of X-linked ichthyosis: identification of a novel mutation (E560P) of the steroid sulfatase gene. Hum Mutat 2000, 15(3):296.

13. Hazan C, Orlow SJ, Schaffer JV: X-linked recessive ichthyosis. Dermatol Online J 2005, 11(4):12.

14. Elias PM, Williams ML, Choi EH, Feingold KR: Role of cholesterol sulfate in epidermal structure and function: lessons from X-linked ichthyosis. Biochim Biophys Acta 2014, 1841(3):353-361.

15. Baek WS, Aypar U: Neurological Manifestations of X-Linked Ichthyosis: Case Report and Review of the Literature. Case Rep Genet 2017, 2017:9086408.

16. Kent L, Emerton J, Bhadravathi V, Weisblatt E, Pasco G, Willatt LR, McMahon R, Yates JR: X-linked ichthyosis (steroid sulfatase deficiency) is associated with increased risk of attention deficit hyperactivity disorder, autism and social communication deficits. J Med Genet 2008, 45(8):519-524.

17. Chatterjee S, Humby T, Davies W: Behavioural and Psychiatric Phenotypes in Men and Boys with XLinked Ichthyosis: Evidence from a Worldwide Online Survey. PLoS One 2016, 11(10):e0164417.

18. Ben Khelifa H, Soyah N, Ben-Abdallah-Bouhjar I, Gritly R, Sanlaville D, Elghezal H, Saad A, MougouZerelli S: Xp22.3 interstitial deletion: a recognizable chromosomal abnormality encompassing 
VCX3A and STS genes in a patient with X-linked ichthyosis and mental retardation. Gene 2013, 527(2):578-583.

19. Freiberg RA, Choate KA, Deng H, Alperin ES, Shapiro LJ, Khavari PA: A model of corrective gene transfer in X-linked ichthyosis. Hum Mol Genet 1997, 6(6):927-933.

20. Ohyama A, Nakano H, Imanishi Y, Seto T, Tsuruta D, Fukai K: A novel missense mutation of the STS gene in two siblings with X-linked ichthyosis, complicated by short stature, bone density reduction, epilepsy, and cryptorchidism. Clin Exp Dermato/ 2019, 44(1):78-79.

21. del Refugio Rivera Vega M, Murillo-Vilches MR, Toral-Lopez J, Sanchez EG, Sanchez AT, GonzalezHuerta LM, Cuevas-Covarrubias SA: X-linked ichthyosis in a patient with a novel nonsense mutation in the STS gene. J Dermato/ Sci 2015, 80(2):160-162.

22. Winge MC, Hoppe T, Lieden A, Nordenskjold M, Vahlquist A, Wahlgren CF, Torma H, Bradley M, Berne B: Novel point mutation in the STS gene in a patient with X-linked recessive ichthyosis. $J$ Dermatol Sci 2011, 63(1):62-64.

23. Afzal S, Ramzan K, Ullah S, Wakil SM, Jamal A, Basit S, Waqar AB: A novel nonsense mutation in the STS gene in a Pakistani family with X-linked recessive ichthyosis: including a very rare case of two homozygous female patients. BMC Med Genet 2020, 21(1):20.

24. Diociaiuti A, El Hachem M, Pisaneschi E, Giancristoforo S, Genovese S, Sirleto P, Boldrini R, Angioni A: Role of molecular testing in the multidisciplinary diagnostic approach of ichthyosis. Orphanet $J$ Rare Dis 2016, 11:4.

25. Liao H, Waters AJ, Goudie DR, Aitken DA, Graham G, Smith FJ, Lewis-Jones S, McLean WH: Filaggrin mutations are genetic modifying factors exacerbating X-linked ichthyosis. J Invest Dermato/ 2007, 127(12):2795-2798.

26. Takeichi T, Sugiura K, Hsu CK, Tanahashi K, Takama H, Simpson MA, McGrath JA, Akiyama M: Novel indel mutation of STS underlies a new phenotype of self-healing recessive X-linked ichthyosis. $J$ Dermatol Sci 2015, 79(3):317-319.

27. Basler E, Grompe M, Parenti G, Yates J, Ballabio A: Identification of point mutations in the steroid sulfatase gene of three patients with X-linked ichthyosis. Am J Hum Genet 1992, 50(3):483-491.

28. Morita E, Katoh O, Shinoda S, Hiragun T, Tanaka T, Kameyoshi Y, Yamamoto S: A novel point mutation in the steroid sulfatase gene in X-linked ichthyosis. J Invest Dermato/ 1997, 109(2):244245.

29. Valdes-Flores M, Vaca AL, Rivera-Vega MR, Kofman-Alfaro SH, Cuevas-Covarrubias SA: Maternal transmission of the 3 bp deletion within exon 7 of the STS gene in steroid sulfatase deficiency. $J$ Invest Dermatol 2001, 117(4):997-999.

30. Oyama N, Matsuda M, Hamada T, Numata S, Teye K, Hashimoto T, Hasegawa M: Two novel missense mutations of STS gene underlie X-linked recessive ichthyosis: understanding of the mutational and structural spectrum. J Eur Acad Dermatol Venereol 2016, 30(9):1629-1631.

31. Wei X, Ju X, Yi X, Zhu Q, Qu N, Liu T, Chen Y, Jiang H, Yang G, Zhen R et al: Identification of sequence variants in genetic disease-causing genes using targeted next-generation sequencing. PLOS One 
2011, 6(12):e29500.

32. Oyama N, Satoh M, Iwatsuki K, Kaneko F: Novel point mutations in the steroid sulfatase gene in patients with X-linked ichthyosis: transfection analysis using the mutated genes. J Invest Dermatol 2000, 114(6):1195-1199.

33. Murtaza G, Siddiq S, Khan S, Hussain S, Naeem M: Molecular study of X-linked ichthyosis: report of a novel 2-bp insertion mutation in the STS and a very rare case of homozygous female patient. $J$ Dermatol Sci 2014, 74(2):165-167.

34. Gonzalez-Huerta LM, Riviera-Vega MR, Kofman-Alfeuro SH, Cuevas-Covarrubias SA: Novel missense mutation (Arg432Cys) in a patient with steroid sulphatase-deficiency. Clin Endocrinol (Oxf) 2003, 59(2):263-264.

35. Trevisson E, Ludwig K, Casarin A, Di Meglio A, Greggio NA, Manara R, Lenzini E, Clementi M, Salviati L: Ichthyosis and Kallmann syndrome: not always a contiguous gene syndrome. J Dermatol Sci 2015, 78(2):158-160.

36. Goodwin G, Hawley PP, Miller DT: A Case of HDR Syndrome and Ichthyosis: Dual Diagnosis by Whole-Genome Sequencing of Novel Mutations in GATA3 and STS Genes. J Clin Endocrinol Metab 2016, 101(3):837-840.

\section{Figures}


(A)

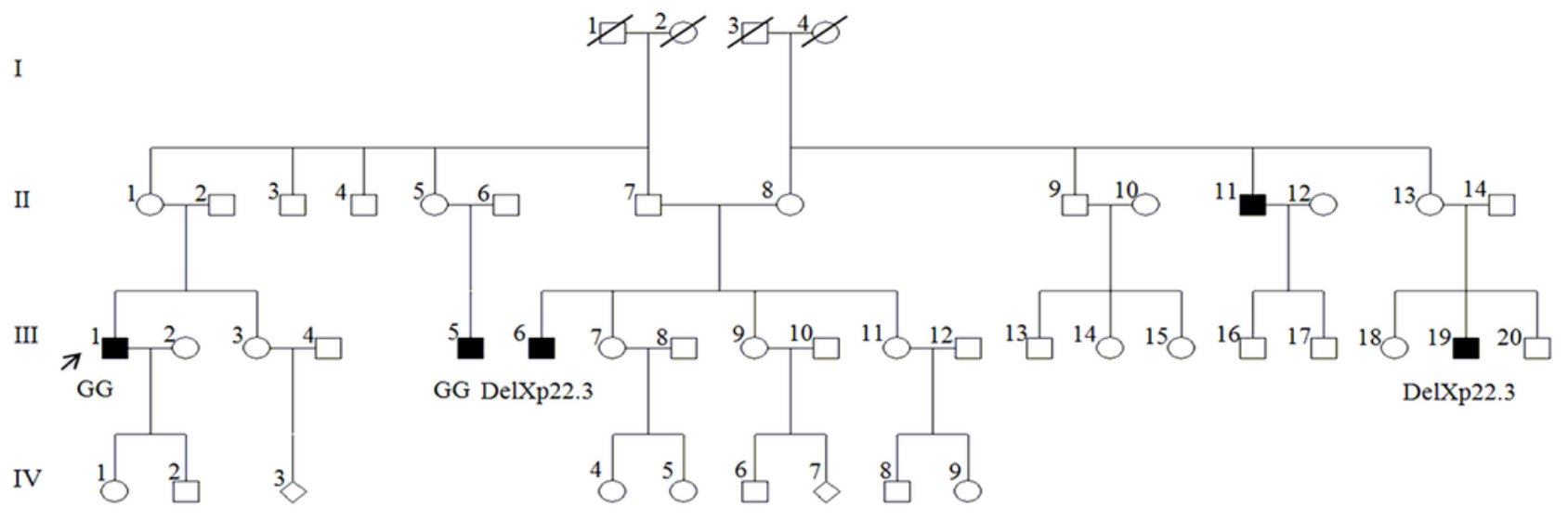

(B)

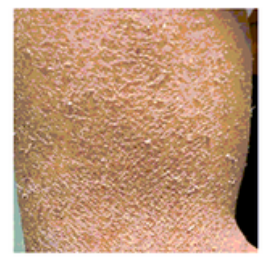

(E)

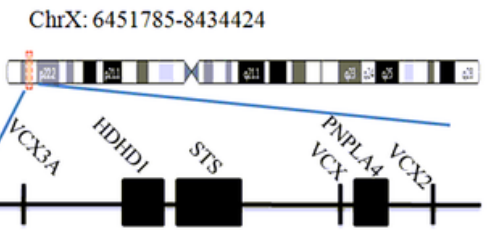

(C)

CCCAGAGGAGAAA

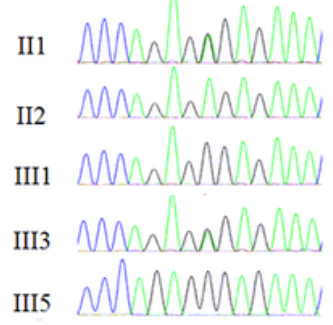

(F)

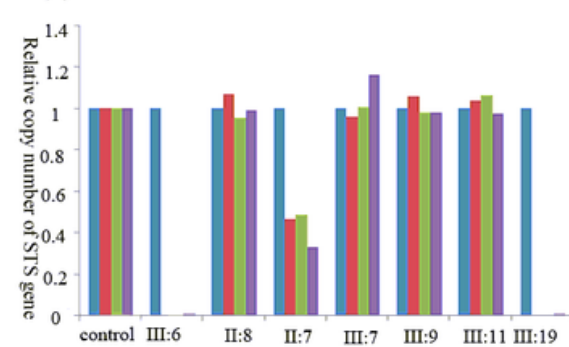

(D)

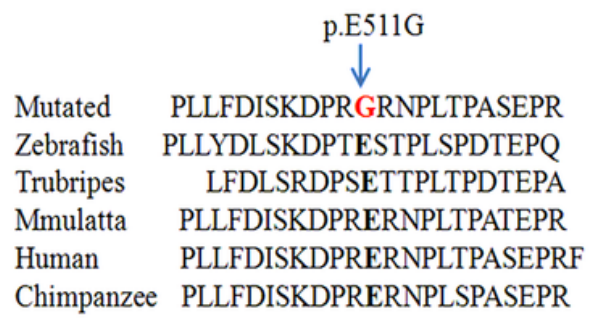

(G)

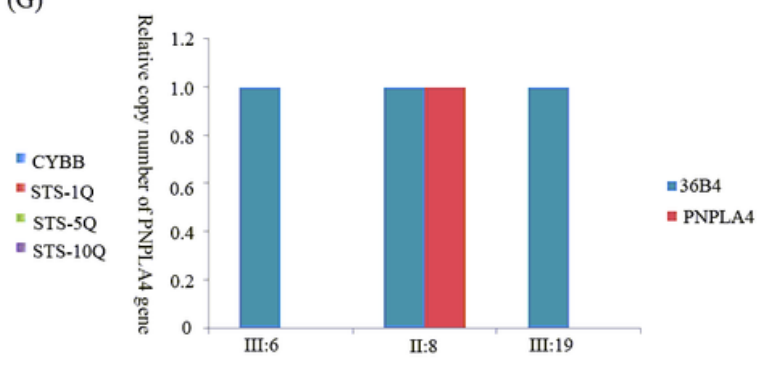

Figure 1

Identification of a novel nonsense mutation in STS gene and a microdeletion in Xq22.3 in a larger family with ichthyosis. (A) The affected members with XLI are depicted by a shaded black square (male). Generations are shown as I to IV. The proband (III:1) is indicated by an arrow. (B) Skin changes of proband (III:1) in spring (left) and in III:6 in winter (right), respectively. (C) Sanger sequencing to confirm the STS c.1532A>G mutation in the proband (III:1), affected member (III:5), and the unaffected control (II:1, II:2, III3). The mutation is marked by an arrow. (D) Conservation analysis of p.E511G in STS across species. (E) RT-PCR to confirm the STS deletion in the affected member (III:6, III:19) and the unaffected control (II:7, II:8,III:7, III:9, III:11), RT-PCR of three exons of STS genes. (F) RT-PCR to confirm PNPLA4 another gene in Xp22.311 deletion in the affected member (III:6, III:19) and the unaffected control ( II:8), RT-PCR of one exons of PNPLA4 genes. (G) Schematic representation of the Xp22.31 region ideogram and some relevant genes of the region (according to UCSC). 


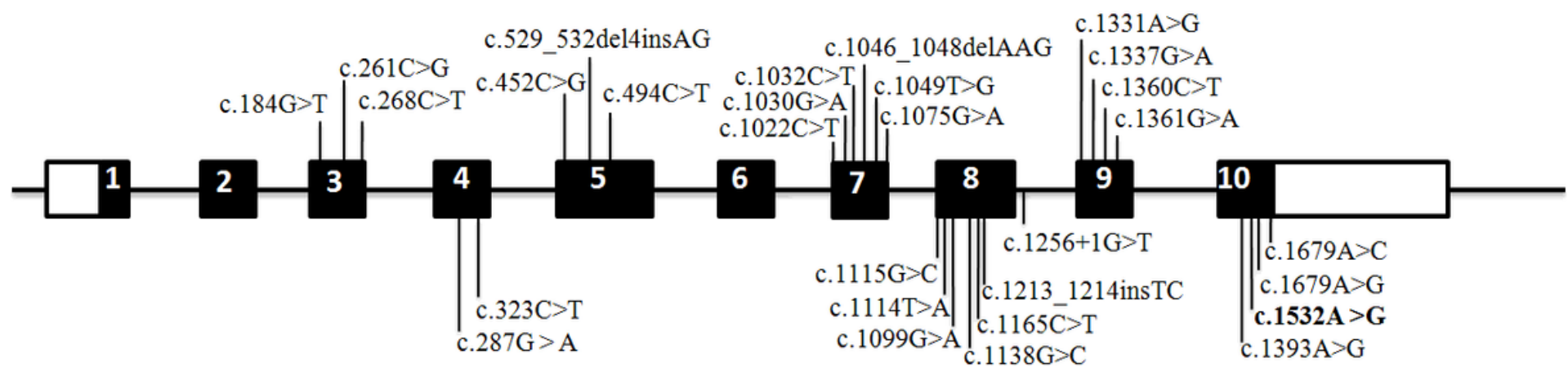

Figure 2

Identified mutations in STS gene in literature and in our study. The mutation in bold was reported in this study. 